\title{
Rare Case of Spontaneous Perinephric Hematoma with Two-Year Follow-Up
}

\author{
Lit Kiat Tan ${ }^{*}$, George Eng Geap Lee ${ }^{2}$ \\ ${ }^{1}$ International Medical University, Bukit Jalil, Malaysia \\ ${ }^{2}$ Monash University Sunway Campus, Bandar Sunway, Malaysia \\ Email: *litkiat_tan@hotmail.com
}

How to cite this paper: Tan, L.K. and Lee, G.E.G. (2020) Rare Case of Spontaneous Perinephric Hematoma with Two-Year Follow-Up. Open Journal of Urology, 10, 233238.

https://doi.org/10.4236/oju.2020.108027

Received: May 26, 2020

Accepted: August 7, 2020

Published: August 10, 2020

Copyright $\odot 2020$ by author(s) and Scientific Research Publishing Inc. This work is licensed under the Creative Commons Attribution International License (CC BY 4.0).

http://creativecommons.org/licenses/by/4.0/ (c) (i) Open Access

\begin{abstract}
Background: Spontaneous perinephric hematoma with no associated pathology or provocation is a rare clinical phenomenon. The hematoma requires a two-year interval for a favorable hematoma resolution, and no associated hypertension or renal scarring. Aims: Evidence of the efficacy of conservative management for spontaneous perinephric hematoma with a 2-year follow up. Case Presentation: A previously healthy 38-year-old woman, presented with a sudden onset of left flank pain, associated with fatigue and pallor. The patient remained hemodynamically stable with no significant history or associated provocations identified. Conclusion: The acute management strategy is favorable in such condition, as the hematoma remains under tamponade in the retroperitoneal space, regardless of the size and organ displacement. Closed observation, serial blood investigation and repeated CT scans are vital to assist in the decision to intervene.
\end{abstract}

\section{Keywords}

Spontaneous Perinephric Hematoma, Gerota's Fascia, Kidney, Lenk’s Triad, Conservative Management

\section{Introduction}

Spontaneous perinephric hematoma was first described in 1856 by Wunderlich, which is defined as the accumulation of blood in the perinephric space from non-traumatic and non-iatrogenic cause [1] [2]. The most common etiology of spontaneous perinephric hematoma is renal angiomyolipoma. The unprovoked spontaneous bleeding from renal angiomyolipoma is observed to correlate with the size of the lesion [3]. Although the spontaneous perinephric bleeding with no associated renal pathology is documented in other literature, its occurrence is 
a rare clinical phenomenon [4]. Regardless of the pathology and the origin of the bleeding, spontaneous perinephric hematoma has clinical manifestation described as Lenk's triad, associated with acute flank pain, flank mass and hypovolemic-shock. The acute and long-term management of the perinephric hemorrhage is also not well reported in other literature. Some clinicians favor exploratory surgery with high possibility of nephrectomy, while others recommended conservative management [5]. Favoring the latter mode of management, we present an unusual case of spontaneous perinephric hematoma effectively treated conservatively. Our case report also has the benefit of progressive follow-up, with regular blood pressure monitoring, ultrasound imaging, urinary analysis and blood investigations. Our data revealed that a favorable resolution of renal hematoma could take up to twenty-four months. The two-year follow-up also has no associated sequelae such as hypertension, renal scaring or functional impairment.

\section{Case Presentation}

A previously healthy 38-year-old woman, presented to the accident and emergency with a sudden onset of left flank pain, with associated fatigue and pallor shortly after the presentation of pain. The patient reported gross hematuria with no associated dysuria. The medical history revealed no evidence of provocation leading to the acute pain. The abdominal examination demonstrated tender mass arising from the left upper quadrant of the abdomen, and the patient remained hemodynamically stable. Laboratory investigation demonstrated anemia (serum hemoglobin at $8.1 \mathrm{~g} / \mathrm{dL}$, Low MCV at $65 \mathrm{fL}$, and Low MCH at $20 \mathrm{pg}$ ) with no impairment of renal function. The urinary analysis confirmed hematuria with no other abnormalities. Abdominal ultrasound revealed a large heterogeneous lesion, measuring $9.8 \mathrm{~cm} \times 6.9 \mathrm{~cm} \times 7.7 \mathrm{~cm}$, located at the left suprarenal region Figure 1.

The initial non-contrasted Computed Tomography Urogram (CTU) confirmed the lesion with internal hyperdense signal, consistent with perinephric hematoma.

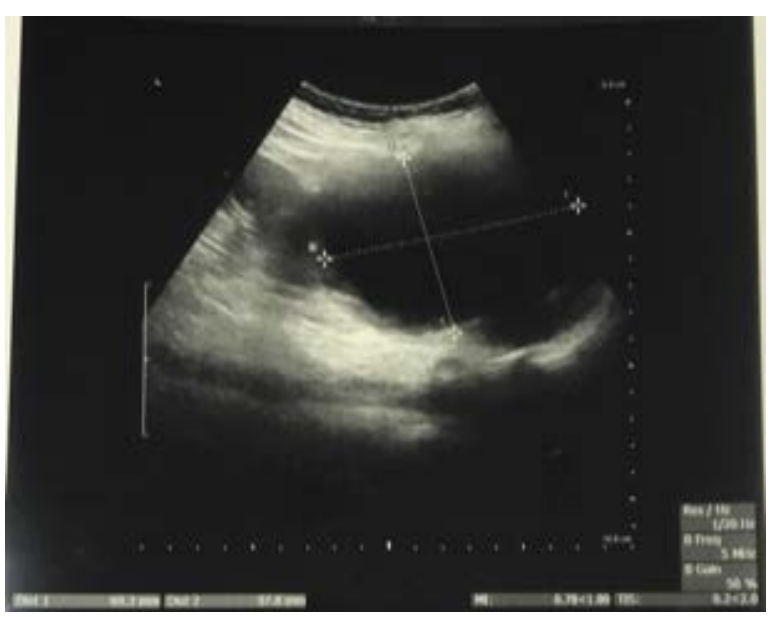

Figure 1. Initial abdominal ultrasound scan $(9.8 \mathrm{~cm} \times 6.9 \mathrm{~cm} \times 7.7 \mathrm{~cm})$. 
The location of the lesion was noted to displace the pancreas superiorly and the left kidney inferiorly Figure 2. The subsequent contrasted CT scan did not demonstrate active hemorrhage; however, the origin of the bleeding was suspected to be arising from the left adrenal resulting in perinephric hematoma in the superior aspect of the left kidney. The patient was admitted for close observation. Patient was on normal saline during admission. Patient is given NSAIDs (IM Pethidine $75 \mathrm{mg}$ PRN) for pain relief and IV prophylactic antibiotic (Ceftriaxone $1 \mathrm{~g} \mathrm{BD}$ ). Four-hourly vital sign examinations were performed, twice daily serial renal function and hemoglobin were also carried out. The renal function profile remained within normal range throughout the admission of the patient, while hemoglobin remained low, ranging from $8.1-8.3 \mathrm{~g} / \mathrm{dL}$. Serial abdominal ultrasound scans were performed at day two and day five following the incident, each with insignificant changes. The patient was discharged uneventfully after she remained pain-free and hemodynamically stable. She was advised to adhere to strict bed rest for a fortnight and daily self-monitoring of the blood pressure. The hematoma remained unaltered. The patient was subsequently followed up with three-monthly abdominal ultrasound scans and laboratory investigations. The two-year follow-up ultrasounds demonstrated significant resolution of the hematoma, with no intra-cystic echogenicity Figure 3. The dimensional

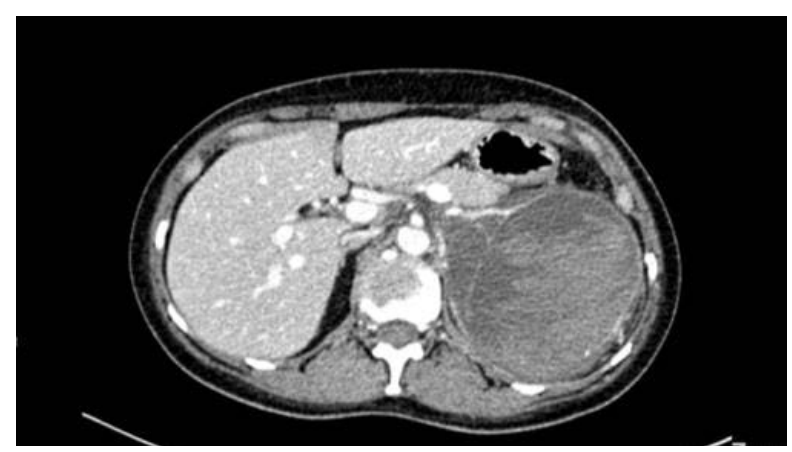

Figure 2. CT Urogram of the Perinephric Hematoma.

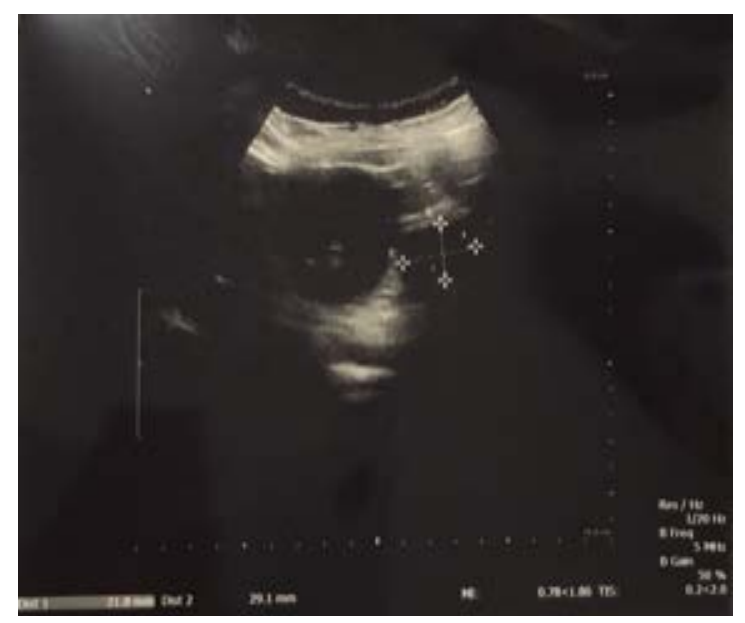

Figure 3. Ultrasound scan showing the resolution of the hematoma. $(2.2 \mathrm{~cm} \times 2.9 \mathrm{~cm} \times 2.0$ $\mathrm{cm})$. 
change of $9.8 \mathrm{~cm} \times 6.9 \mathrm{~cm} \times 7.7 \mathrm{~cm}$ to $2.2 \mathrm{~cm} \times 2.9 \mathrm{~cm} \times 2.0 \mathrm{~cm}$ was calculated, which translated to a volume reduction from $84.8 \mathrm{cc}$ to $10.57 \mathrm{cc}$ over a twenty-four-month interval.

\section{Discussion}

Spontaneous perinephric hematoma is uncommon. However, the initial management of such condition is critically important, as the acute uncontrolled bleeding can be life threatening. The mode of intervention is generally dependent on factors such as the symptomology and hemodynamic stability. Multi-phased contrasted CT scan is the goal standard to determine the extent of a hematoma, adjacent organs involvement and the source of bleeding [1]. Repeated CT scans may also be necessary, as the secondary bleeding can also change the course of intervention. The overall patient symptoms, hemodynamic stability and the extent of injury on CT scan are important factors to take into account prior to determining the need for conservative management, radiological intervention or surgical exploration [2].

According to the literature review, the recommendation for the initial management of spontaneous perinephric hematoma is variable. One study recommended exploratory surgery and nephrectomy for all cases, regardless of patient's status and the severity of hematoma. Such decision was based on the observation that $43 \%$ of the individuals were diagnosed to have had renal malignancy after the nephrectomy. Therefore, the authors from the study advocated nephrectomies for all sufferers of spontaneous perinephric hematoma [5]. Another publication supported conservative management as a first line treatment. The study recommended close observation as the primary protocol in yielding a successful outcome. However, additional intervention may be necessary dependent on the etiology of the hematoma [6]. Patients with functional impairment due to compression or other pathology associated with spontaneous hematoma would require emergency surgical intervention namely nephrectomy or percutaneous drainage. The monitoring protocol after the spontaneous perinephric hematoma is also reported. Following the initial conservative management, abdominal ultrasound scans at regular intervals beyond eighteen months was advocated [7].

We present a rare case of spontaneous bleed with no associated provocation or renal pathology. To our best knowledge, such presentation of perinephric hematoma is unreported in other literature. In most instances, the initial conservative management is favorable in such condition. Anatomically, active bleeding often ceases when the hematoma remains under tamponade in the retroperitoneal space, regardless of the size and organ displacement. Closed observation, serial blood investigation and repeated CT scans are useful guides on decisions to intervene. Our patient also benefited from bed rest and prophylactic antibiotics for a fortnight, as such measures minimize the risk of secondary bleed and infections in the initial stages of recovery.

The close monitoring of patients following the acute phase management is al- 
so necessary. We also present the longest duration of a follow-up in literature. Apart from monitoring the regression of the hematoma, the follow-up consultations also ensured no complications of perinephric abscess, hypertension or renal scaring. Blood and urine investigations are also helpful to ensure overall recovery. Our serial imaging revealed no changes are expected within three weeks following the initial onset of the bleeding. Our longest follow-up of such injury showing eighty percent resolution of the hematoma may require a two-year interval. Lastly, no demonstrable complications of renal scarring or associated hypertension are to be expected. Notably the case report is revealing clinical experience of a single patient, however the rarity of non-provocated bleeding and the relatively long interval of follow-up will provide important data for future management of spontaneous perinephric hematoma.

\section{Conclusion}

The initial management of spontaneous perinephric hematoma is important as acute uncontrolled bleeding can be life threatening. The acute management strategy in this case has been proven to be favorable, as the hematoma remained under tamponade in the retroperitoneal space regardless of the size and the displacement of other organs. Close observation, serial blood investigation, and CT scans are vital to assist in the decision to intervene. The conservative management of the patient followed by 2 years of close monitoring with regular blood pressure monitoring, ultrasound imaging, urinary analysis, and blood investigations has benefited the patient with the eventual resolution of the hematoma without any complication. Due to the rarity of non-provocation bleeding, we believe the information presented here will benefit future managements of spontaneous perinephric hematoma.

\section{Statement of Informed Consent}

A verbal informed consent was obtained from the patient for the information and images used in this publication.

\section{Conflicts of Interest}

The authors declare no conflicts of interest regarding the publication of this paper.

\section{References}

[1] Mao, Y., De Oliveira, I.S., et al. (2017) Etiology, Imaging Features, and Evolution of Spontaneous Perirenal Hemorrhage. Clinical Radiology, 72, 175.e19-175.e26. https://doi.org/10.1016/j.crad.2016.08.010

[2] Liu, L., et al. (2018) A Preliminary Study on Classification and Therapeutic Strategies for. International Journal of Surgery, 54, 86-91. https://doi.org/10.1016/j.ijsu.2018.04.029

[3] La, F.S., et al. (2016) Clinical Management of Spontaneous Perirenal Hematomas without Renal Causes: A New Urological Challenge. European Urology Supplements, 
15, e134. https://doi.org/10.1016/S1569-9056(16)60136-7

[4] Ahn, T., et al. (2017) Changing Etiology and Management Patterns for Spontaneous Renal Hemorrhage. International Urology and Nephrology, 49, 1897-1905. https://doi.org/10.1007/s11255-017-1694-8

[5] Guttilla, A., et al. (2013) Wunderlich's Syndrome: Three Cases of Acute Spontaneous Renal Bleeding, Conservatively Treated. Archivio Italiano Di Urologia E Andrologia, 85, 210-213. https://doi.org/10.4081/aiua.2013.4.210

[6] Zhang, J.Q., et al. (2002) Etiology of Spontaneous Perirenal Hemorrhage: A Meta-Analysis. Journal of Urology, 167, 1593-1596.

[7] Shih, W.J. (2000) Spontaneous Subcapsular and Intrarenal Hematoma Demonstrated by Various Diagnostic Modalities and Monitored by Ultrasonography until Complete Resolution. Journal of the National Medical Association, 92, 200-205. 